STRUCTURAL SCIENCE CRYSTAL ENGINEERING MATERIALS

ISSN 2052-5206

Received 21 May 2021

Accepted 21 May 2021

Keywords: radiation damage; molecular crystal; phase transition; thermal expansion; singlecrystal diffraction.

\section{Impact of synchrotron X-ray radiation damage on a molecular ferroelectric crystal}

\author{
Ines E. Collings*
}

Centre for X-ray Analytics, Swiss Federal Laboratory for Materials Science an Technology (Empa), Überlandstrasse 129, Dübendorf, CH-8600, Switzerland. *Correspondence e-mail: collings.ie@gmail.com

With the increasing use of third- and fourth-generation synchrotrons, the small-molecule crystallographic community has recently begun to assess the radiation damage impact of high intensity beams on samples. This is especially important for in situ studies, where the material of interest is investigated as a function of various external conditions and measured multiple times. If the sample is changing not only as a function of applied stimulus, e.g. temperature or pressure, but also with the duration of X-ray irradiation, how can these two effects be separated? Furthermore, if the sample does exhibit radiation damage, will this reduce or enhance material functionality; in other words, could we begin to use radiation to fine-tune properties on the micron to nanoscale?

In the article by Bogdanov et al. (2021) published in this issue, the authors tackle these questions with their study on the molecular ferroelectric crystal glycinium phosphite (GPI), which has the chemical formula $\mathrm{NH}_{3} \mathrm{CH}_{2} \mathrm{COOH} \cdot \mathrm{H}_{2} \mathrm{PO}_{3}$. At the European Synchrotron Radiation Facility (ESRF), they performed variable-temperature singlecrystal diffraction measurements in fine-temperature steps to fully characterize the order parameters and strains involved in the ferroelectric transition of GPI. Since the ferroelectric behaviour is associated with an ordering of light hydrogen atoms and rearrangement of hydrogen bonds, single-crystal diffraction is favoured over the faster powder diffraction measurements. However, a deterioration of the data quality is observed upon cooling, manifested by a suppression of scattering at high angles and an increase of atomic displacement parameters for all atoms. Since this suggests the possibility of radiation damage, the authors complemented their synchrotron study with a similar experiment using a laboratory diffractometer. Most striking is the completely different thermal expansion response of GPI upon cooling. In the laboratory experiment, a volume contraction of $1.2 \%$ is observed upon cooling to $100 \mathrm{~K}$, in contrast to the volume expansion of $0.4 \%$ recorded in the synchrotron study. Thus, two effects are acting on the GPI crystal in the synchrotron study: the first is the influence of cooling, and the second, more dominant, impact is that of the radiation damage. Even though the effects of radiation damage are reduced at low temperatures (Goeta \& Howard, 2004), the GPI crystal exhibits a steady increase in volume that is correlated to the duration of synchrotron X-ray irradiation.

The X-ray-induced volume expansion in GPI is thought to be related to the formation of $\mathrm{PO}_{3}{ }^{2-}$ radical defects (Vanishri et al., 2007). However, such structural defects are hard to identify using X-ray crystallography since defective-GPI and GPI would only differ in the occupation of hydrogen atoms. Indeed, despite the discrepancy in the unit-cell dimensions, the structural models show very similar atomic positions in both synchrotron and laboratory data sets. Moreover, Bogdanov et al. (2021) show that the paraelectric to ferroelectric transition temperature of GPI at $225 \mathrm{~K}$ remains unchanged for both GPI crystals measured at the synchrotron and in the laboratory. Further studies are needed to assess whether defective molecular ferroelectrics, with enlarged volumes from X-ray irradiation, would give rise to new functionality.

Whether we seek to avoid or utilize radiation damage in our structural research, what is clear is that a greater understanding of its impact and ways to control it are needed. Already, we are heading in this direction, with a greater number of systematic studies on radiation damage (Christensen et al., 2019). However, the diversity in small-molecular systems makes it very challenging to propose general guidelines for radiation impact, as is documented in the field of macromolecular crystallography (Garman \& Weik, 2017; Garman \& Weik, 2019). While synchrotron beamtimes will likely remain dedicated to 


\section{scientific commentaries}

understanding the material and its functionality, additional good practices can be implemented to monitor the role that radiation can play. This can include several re-measurements on the same sample position and conditions after a given time of irradiation, in addition to measurements on different sample positions that were not exposed to the beam, as was performed in the investigation by Coates et al. (2021). Another strategy, as highlighted by Bogdanov et al. (2021), is the use of complementary X-ray laboratory or neutron data, where the radiation damage is substantially less than that from synchrotron sources, to disentangle the effects of radiation damage and material behaviour.

\section{References}

Bogdanov, N., Zakharov, B., Chernyshov, D., Pattison, P. \& Boldyreva, E. (2021). Acta Cryst. B77, 365-370.

Christensen, J., Horton, P. N., Bury, C. S., Dickerson, J. L., Taberman, H., Garman, E. F. \& Coles, S. J. (2019). IUCrJ, 6, 703-713.

Coates, C. S., Murray, C. A., Boström, H. L. B., Reynolds, E. M. \& Goodwin, A. L. (2021). Mater. Horiz. 8, 1446-1453.

Garman, E. F. \& Weik, M. (2017). J. Synchrotron Rad. 24, 1-6.

Garman, E. F. \& Weik, M. (2019). J. Synchrotron Rad. 26, 907-911.

Goeta, A. E. \& Howard, J. A. K. (2004). Chem. Soc. Rev. 33, 490-500.

Vanishri, S., Reddy, J. N. B. \& Bhat, H. L. (2007). J. Appl. Phys. 101, 054106. 